\title{
Application of the VORTFIND algorithm for the identification of vortical flow features around complex 3D geometries
}

\author{
A.B. Phillips*and S.R. Turnock \\ Fluid Structure Interactions Research Group, University of Southampton, SO17 1BJ, UK
}

\section{SUMMARY}

Accurate prediction of the hydrodynamic forces and moments acting on a manoeuvring marine vehicle using Reynolds Averaged Navier Stokes (RANS) simulations requires sufficient mesh resolution to capture offbody vortical structures. Since the path of these structures is not known a priori, a vortex identification and capture strategy is required alongside an iterative mesh adaption process. An improved version of the VORTFIND algorithm which can identify multiple vortices of variable strength and rotational direction using a K-means algorithm is described. The algorithm is applied to velocity fields generated from RANS simulations to increase the mesh resolution in the vortex core region, ensuring sufficient mesh density to capture the downstream propagation of the vortex for a submarine hull at drift and ship propeller-rudder interaction. Copyright (c) 2011 John Wiley \& Sons, Ltd.

Received ...

KEY WORDS: vortex identification; adaptive mesh refinement; RANS; ship hydrodynamics

\section{INTRODUCTION}

The hydrodynamic forces and moments acting on a manoeuvring vessel are a result of the complex interaction between the viscous fluid and the hull. The use of Reynolds Averaged Navier Stokes (RANS) simulations to derive the hydrodynamic derivatives for ships and submarines

${ }^{*}$ Correspondence to: Fluid Structure Interactions Research Group, University of Southampton, SO17 1BJ, UK. 
consistently show significant discrepancies between the numerical and experimental prediction of the hydrodynamic moments [1, 2, 3, 4]. One of the contributing factor to this, is the poor resolution of vortical structures generated by the hull and control surfaces. Simulations [5, 6] show that improved agreement with experimental results may be obtained by locally refining the vortex core region.

Capturing off body vortex structures requires that both the vortex formation and downstream evolution of the vortex are adequately captured. Insufficient mesh elements in the vortex core region leads to significant numerical diffusion which artificially dissipates the vortex. This can have a significant impact on longitudinal pressure distribution of downstream bodies with which the vortex interacts. Correct capture of the low pressure region associated with the tip vortex core is also important when considering downstream propagation of cavitation [7, 8] . It is worth highlighting that for many typical flows around marine vehicles the user has sufficient elements to resolve the boundary layer and off body vortex structures to a level where selection of turbulence model also has a significant impact on the downstream evolution of the vortex. Selection of standard isotropic two equation models leads to more rapid dissipation of vortical structure, compared to Reynolds Stress Models [9]. The generation of a suitably efficient, high quality mesh is not a simple task since the path, strength and radius of the vortex is not known a priori.

This paper describes and extends the VORTFIND algorithm to robustly identify an initially unknown number of multiple vortices of variable strength and direction generated around a manoeuvring vehicle. Two distinct applications of the use of the improved VORTFIND method in conjunction with Reynolds Averaged Navier Stokes (RANS) simulations are demonstrated; body vortex path along the hull of a manoeuvring submarine and multiple vortex identification in the idealised flow behind a propeller and rudder.

\section{VORTEX IDENTIFICATION}

In order to achieve an iterative improvement in mesh resolution at the location of vortical structures, a suitable vortex identification method is required. Summaries of available identification methods 
are provided by [10, 11]. Jiang et al. [10] divides the methods into three taxonomies. The first taxonomy identifies whether the vortex is treated as a region or as a line, the second queries whether the method is Galilean invariant, and the final taxonomy classes detection methods based on whether they use local cell neighbour information. Methods available include: Q-criterion [12], Helicity Method, [13], Swirl Parameter Method, [14], Lambda2 Method [15], Predictor-Corrector Method [16], Eigenvector Method [17], Parallel Vectors Method [18], Maximum Vorticity Method [19], Streamline Methods [20] and the Combinatorial Method [21].

The original VORTFIND algorithm developed by Pemberton et al. [22] was inspired by the definition of a vortex as described by Lugt [23], 'A vortex is the rotating motion of a multitude of material particles around a common centre', to identify vortex core centres using just the velocity data on a transverse planes. The original VORTFIND methodology is detailed below:-

1. For all inspection points on a plane perpendicular to the vortex direction, the angle $\alpha$ between the transverse velocity and a reference axis on the plane is calculated. An integer index $\beta$ is used to classify in which of $n$ velocity direction sectors the point lies, Table I. Figure 1 illustrates the classification process for $n=3$.

Table I. $\beta$ Values for $\mathrm{n}$ sectors.

\begin{tabular}{|c|c|}
\hline Transverse flow direction, $\alpha(\mathrm{deg})$ & Sector index, $\beta$ \\
\hline $0<\alpha \leq 360^{\circ} / n$ & 0 \\
$360^{\circ} / n<\alpha \leq 360^{\circ} / n \times 2$ & 1 \\
$\ldots$ & $\ldots$ \\
$360^{\circ} / n \times(n-1)<\alpha \leq 360^{\circ}$ & $\mathrm{n}-1$ \\
\hline
\end{tabular}




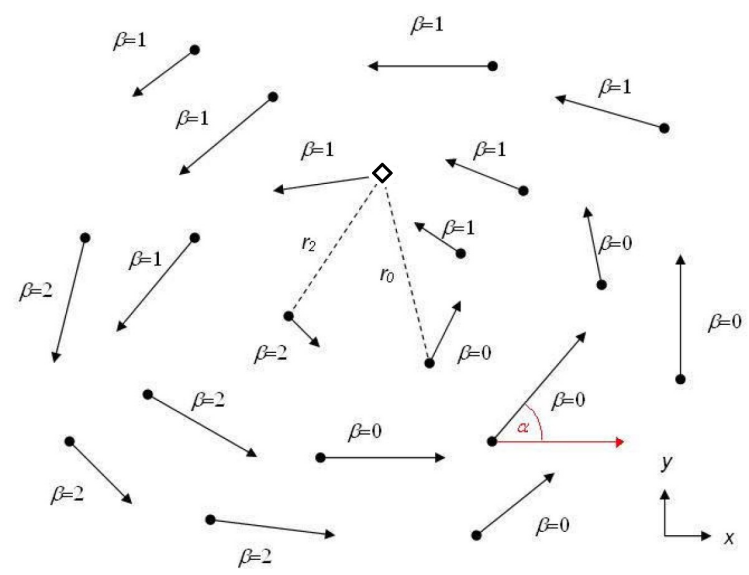

Figure 1. Schematic representation for calculating $\ln$, for three $\operatorname{sectors}(n=3) . \diamond$ reference point, $\bullet$ test points.

2. For each reference point a vector $r_{i}$ of dimension $\mathrm{n}$ is evaluated based on the distance to the nearest point in each sector $\beta$. For the example in Figure 1 with three sectors $r_{1}$ is zero, similarly for sectors where no point is found a value of 0 is applied.

3. The scalar $l n$ is then calculated for each point,

$$
l_{n}=\frac{\sum_{i=0}^{n-1} r_{i}^{2}}{n_{\text {Bexist }}}
$$

where $n_{\text {Bexist }}$ is the number of populated sectors.

4. The centre of the vortex will lie nearest to the point with the minimum $l_{n}$.

This method may be extended to tracking vortices in three dimensions by using a series of planes that are close to normal to the local vortex direction [24]. A vortex core line is generated using a series of line segments passing through the local minima of the series of planes. It is classified as a line method that although not Galilean invariant allows multiple vortices to be captured. It only requires knowledge of the velocity field, as opposed to the methods which require further details such as grid connectivity or velocity gradients and exhibit poor performance on an initial coarse mesh required to initiate the mesh adaptation cycle. 
In order to identify the vortex centre the VORTFIND algorithm must be performed on a plane close to perpendicular $( \pm 30 \mathrm{deg})$ to the path of the vortex core. The majority of vortex structures around manoeuvring marine vehicles are typically convected downstream along paths not dissimilar to the inflow conditions, consequently selection of planes perpendicular to the inlet velocity vector are typically appropriate. There are exceptions, for example identification of the initiation of the horseshoe vortex at a hull-control surface junction. In this case a second approach can be adopted which uses an alternative reference direction.

The original VORTFIND method has been successfully used on numerical data sets to identify: bilge vortices [25], control surface tip vortices [24] and propeller tip vortices [5]. It has also been used on experimental particle image velocimetry (PIV) data to track the transient path of tip vortex downstream of rudder undergoing periodic motion [26]. However, when applied to complex 3D geometries, limitations to the algorithm where identified in flows with multiple vortices, which occasionally lead to false returns at turning and saddle points, see section 4 .

\section{IMPROVED VORTFIND ALGORITHM}

For more complex flows around manoeuvring vehicles, modifications to the VORTFIND algorithm are required to correctly identify multiple vortex structures of differing strength and direction. The goals of the improved formulation are to: restrict the algorithm to only analysing the velocity vectors; identify all vortices equally independent of vortex strength; minimise mesh influence on the solution and remove false returns by analysing the direction of rotation.

A more detailed definition of a vortex is chosen as: 'A vortex is the rotating motion of material particles in the same direction about a common centre'. Hence, it is proposed to incorporate the angular velocity into the implementation of the VORTFIND algorithm. In two dimensions the angular velocity, $\omega$, at point $P$ relative to point $O$, is a function of the radius, $\bar{r}=P-O$, and the velocity, $\bar{v}$, at point $P$ :

$$
\omega=\frac{\bar{r} \times \bar{v}}{|\bar{r}|^{2}}
$$


the direction of the angular velocity, $\bar{\omega}$, may be determined from:

$$
\bar{\omega}=\frac{\bar{r} \times \bar{v}}{|\bar{r} \times \bar{v}|},
$$

where by convention a result of 1 corresponds to an anticlockwise rotation, while a result of -1 corresponds to a clockwise rotation.

The modified algorithm still considers a two-dimensional slice of fluid perpendicular to the axis of rotation of the vortex. The methodology to calculate $l_{n}$ is changed such that it considers clockwise and anticlockwise vortices separately:-

1. The velocity data is mapped onto a regular Cartesian mesh $(N x \times N y$ nodes $)$. This reduces mesh dependencies and may be used to reduce total runtime if an appropriate Cartesian mesh size is selected.

2. At each point on the plane, $\alpha$ is calculated, and it's $\beta$ value is classified.

3. Each node is considered in turn. For each of the surrounding nodes, the distance between the principle node under consideration and the surrounding nodes, $r$, is calculated as well as the direction of the angular velocity induced by the velocity vector at the surrounding nodes about the principle node. See Figure 2.

4. Classifying vortex structures is split into considering anticlockwise and clockwise vortices. Firstly, the anticlockwise $l_{n}$ is determined. At each node, the distance to the nearest surrounding node with $\beta$ values of $0,1 \ldots n$ with an angular direction of +1 is calculated and stored for each point as $r_{1}, r_{2} \ldots$ and $r_{n}$. The $l_{n_{\text {anticlockwise }}}$ is then calculated for each node: -

$$
l_{n_{\text {anticlockwise }}}=\frac{\sum_{i=0}^{n-1} r_{i}^{2}}{n_{\text {Bexist }}}
$$

5. Secondly $l_{n_{\text {clockwise }}}$ is determined. At each node, the distance to the nearest surrounding node with $\beta$ values of $0,1 \ldots \mathrm{n}$ with an angular direction of -1 is calculated and stored for each point as $r_{1}, r_{2} \ldots$ and $r_{n}$. The $l_{n_{\text {clockwise }}}$ is then calculated for each node: - 


$$
l_{n_{\text {clockwise }}}=\frac{\sum_{i=0}^{n-1} r_{i}^{2}}{n_{\beta e x i s t}}
$$

6. The final $l n$ is the minima of the clockwise and anticlockwise $l n$ for each node.

$$
l_{n}=\min \left(l_{n_{\text {anticlockwise }}}, l_{n_{\text {clockwise }}}\right)
$$

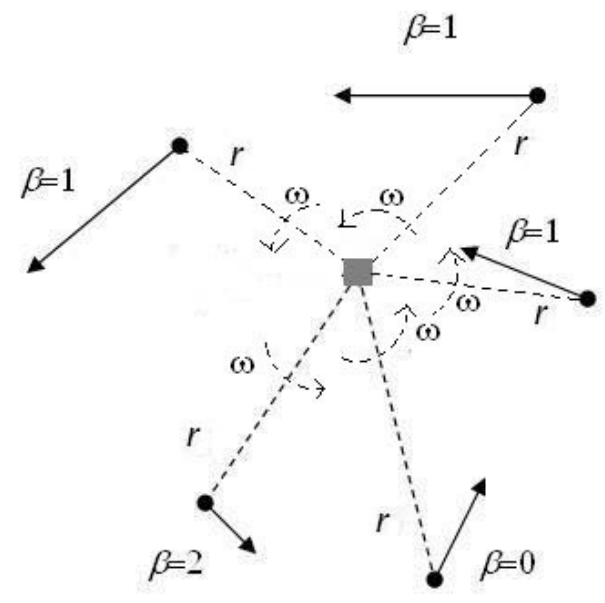

\section{Principle Node}

- Surrounding Nodes

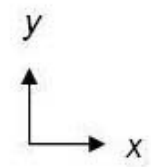

Figure 2. Simplified schematic of the extended VORTFIND algorithm with three sectors. In the current implementation, the surrounding nodes comprise all the nodes on the plane except the principle node.

For flows with only one vortex the node with the lowest $l_{n}$ corresponds to the vortex centre. For many external flows, the number of vortices produced by a body may be an unknown and hence the process of identifying multiple vortex cores on a plane requires local minima in $l_{n}$ to be identified, since on a uniform mesh the magnitude of the $l_{n}$ is independent of vortex strength. Pemberton [27] found that an adaptive K-Means cluster algorithm [28], was appropriate for this case. 
The $m$ nodes with the smallest $l_{n}$ are moved to a subgroup of the data. These data points are assigned to clusters by implicitly minimising $\mathcal{J}$, the sum of the distance squared from the clusters centroid to the points within it,

$$
\begin{gathered}
\mathcal{J}=\sum_{j=1, i=1}^{j=N_{p_{i}}, i=N_{c}-1}\left(x_{j i}-\mu_{x i}\right)^{2}+\left(y_{j i}-\mu_{y i}\right)^{2}, \\
\mu_{x i}=\frac{1}{N_{p_{i}}} \sum_{j=1}^{N_{p i}} x_{j i}, \\
\mu_{y i}=\frac{1}{N_{p_{i}}} \sum_{j=1}^{N_{p i}} y_{j i},
\end{gathered}
$$

where $\mu_{x i}$ and $\mu_{y i}$ are co-ordinates of the centroid centre, $N_{p_{i}}$ is the number of points in the $i^{t h}$ cluster, $N_{c}$ is the number of clusters and $x_{j i}$ and $y_{j i}$ are the co-ordinates of the $j^{t h}$ point in the $i^{t h}$ cluster. Since the number of vortices and hence clusters is not known a priori, the minimal distance between vortices, $d_{\min }$ and the maximum vortex radius must be defined, $r_{\max } . \mathcal{J}$ is minimised using the following procedure, adapted from [28]:-

1. Choose an initial value of $N_{c}$, then take the first $N_{c}$ data points as initial clusters with one member each.

2. Compute the pairwise distance between the first $N_{c}$ data units.

3. If the pairwise distance is less than $d_{\min }$ then merge the two associated clusters and compute the distance between the centroid of the new cluster and all remaining clusters.

4. Continue merging clusters as required until all centroids are separated by a distance at least as large as $d_{\min }$.

5. Assign the remaining data points, $N_{c}+1$ through to $m$, to the nearest centroid and update the cluster centroid if the distance to the nearest centroid is less than $r_{\max }$, or take the data point and create a new cluster with one member. 
6. Compute the pairwise distance between the first $N_{c}$ data units. If the pairwise distance is less than $d_{\min }$ then merge the two associated clusters and compute the distance between the centroid of the new cluster and all remaining clusters.

7. After data units $N_{c}+1$ through $m$ have been assigned, take the existing cluster centroids as fixed seed points and reallocate each data point to its nearest seed point.

The algorithm stops after the first reallocation with no further attempts to improve convergence and thus the computational time is economical. The choice of clustering parameters $d_{\min }$ and $r_{\max }$ will affect the solution. Typically, the order of the size of the tip vortex or body vortex structure may be predicted by knowing the scale size of the body.

For mesh refinement purposes, this level of accuracy is appropriate since by refining the mesh in this region, the path of the vortex is likely to change.

The combined improved VORTFIND and K-Means algorithm approach presented has four control variables which can be tuned to refine the search algorithm to a specific flow:-

1. regular mesh size data is mapped onto $(N x \times N y)$;

2. percentage of data points extracted to use for K-means approach $\left(P_{N}\right)$;

3. maximum vortex radius allowed in K-means algorithm $\left(r_{\max }\right)$;

4. minimum vortex separation allowed in K-means algorithm $\left(d_{\text {min }}\right)$.

\section{DEMONSTRATION OF THE IMPROVED VORTFIND ALGORITHM}

To demonstrate the capability of the improved VORTFIND algorithm to identify multiple vortices a series of simple potential flow cases will be considered. Also presented are results from the original VORTFIND algorithm and the Q-Criterion [12]:

$$
Q=\frac{1}{2}\left(\|\Omega\|^{2}-\|S\|^{2}\right),
$$


where $S_{i j}$ and $\Omega_{i j}$ are strain and rotation tensors defined by $S_{i j}=\left(\partial U_{i} / \partial x_{j}+\partial U_{j} / \partial x_{i}\right) / 2$ and $\Omega_{i j}=\left(\partial U_{i} / \partial x_{j}-\partial U_{j} / \partial x_{i}\right) / 2$. A coherent vortex is defined as a region where $Q>0$. Gradients for the Q-Criterion calculation have been determined via 2nd order central differencing.

Firstly, consider four potential vortices in a diamond configuration about the origin. Each vortex has equal magnitude of circulation strength but alternating direction as shown in Figure 3(a). Taking the resulting velocity field, Figure 3(b), and applying the original VORTFIND algorithm with eight sectors, five minima are identified, four coinciding with the centres of the vortex, and one false return at the origin, Figure 3(c). The false return corresponds to a location which is surrounded by velocity vectors pointing in directions corresponding to the 8 sectors used in this calculation, see inset of Figure 3(b). Consequently, a minima is identified in the $l_{n}$, of the same magnitude as the true vortex locations. However, the surrounding velocity vectors are not all rotating in the same coherent angular direction, and as such this is not a vortex centre.

Using the improved VORTFIND algorithm gives the results shown in Figure 3(e). With the improved formulation the peaks in $l n$ correspond with the defined vortex locations only. Since the direction of the rotation is included in the algorithm, saddle and turning points no longer give false returns of equal magnitude to vortices. The Q-Criterion also provides clear identification of the four vortex structures.

Consider the $2 \mathrm{D}$ problem of two potential vortices, a primary vortex of strength $\Gamma=-10 \mathrm{~m}^{2} / \mathrm{s}$ and weaker secondary vortex $\Gamma=2 \mathrm{~m}^{2} / \mathrm{s}$ located at $(-1,0)$ and $(1,0)$ respectively, and a free stream velocity of $v=1 \mathrm{~m} / \mathrm{s}$, Figure 4 .

Four clear minima are identified by the original VORTFIND method, two correspond to the potential vortex centres, while the two erroneous minima are located at turning points within the flow, see Figure 4(c). Again the improved VORTFIND algorithm removes the erroneous minima just returning the correct vortex centres, Figure 4(d). While the Q-Criterion (Figure 4(e)) also identifies the correct vortex cores the stronger vortex is identified more clearly, due to the higher magnitude of the rotation tensor. 


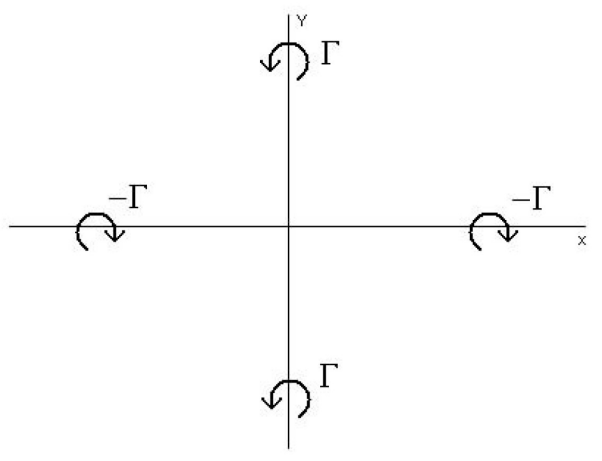

(a) Vortices

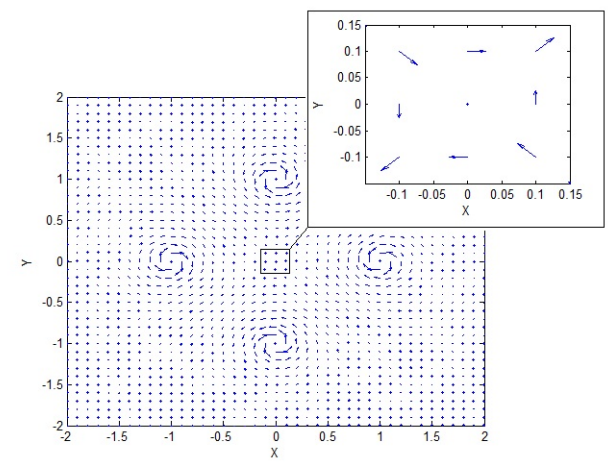

(b) Velocity Vectors

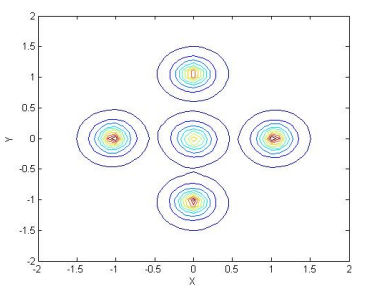

(c) $1 / l_{n}$ original VORTFIND

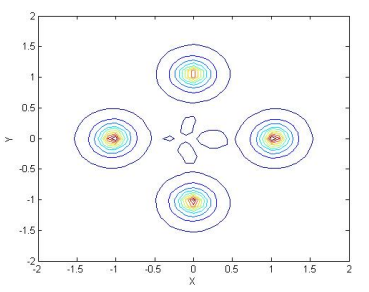

(d) $1 / l_{n}$ improved VORTFIND

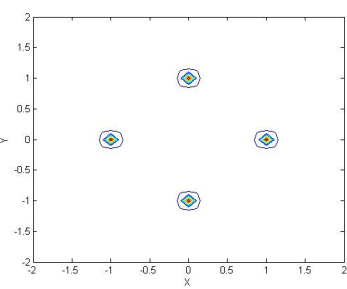

(e) Q-Criterion

Figure 3. Application of the improved VORTFIND algorithm with 8 sectors to four potential vortices in a diamond configuration, grid size $41 \times 41$ nodes.

The greater clarity of the improved VORTFIND classification comes at an additional computational cost. The computational cost is still small compared to the cost of determining the flow field using a higher resolution CFD analysis. The key advantages are the ability to identify vortex structures independent of vortex strength, and since velocity gradients are not required it is simple to apply to unstructured velocity data.

\section{APPLICATION OF VORTFIND TO COMPLEX FLOWS}

To demonstrate the application of the improved VORTFIND algorithm to real flows where the number and path of the vortices are not known a priori, two case studies are presented. Where the improved VORTFIND algorithm is used in conjunction with RANS simulation.

An estimate of the full steady state three dimensional flow field for the two flows considered are determined using the incompressible (11), isothermal Reynolds Averaged Navier Stokes (RANS) 


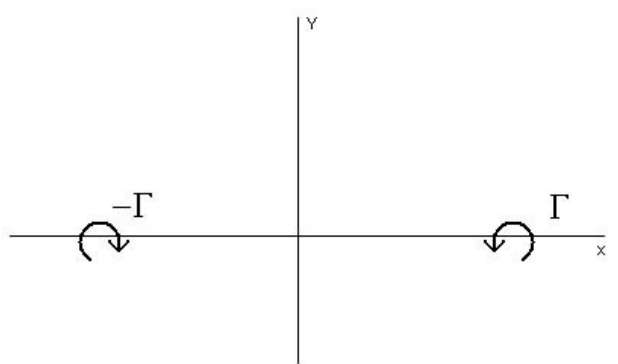

$\uparrow_{\text {(a) Vortices }}^{V}$

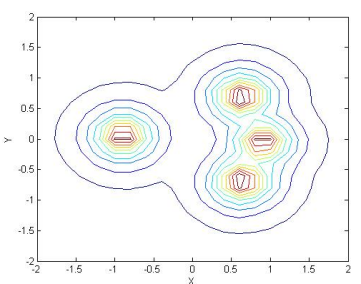

(c) $1 / l_{n}$ original VORTFIND

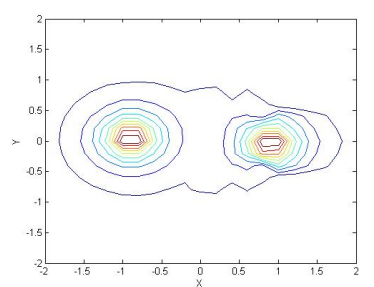

(d) $1 / l_{n}$ improved VORTFIND

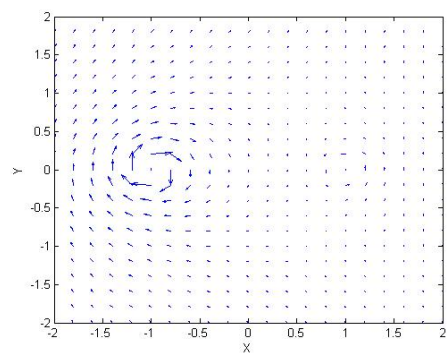

(b) Velocity Vectors

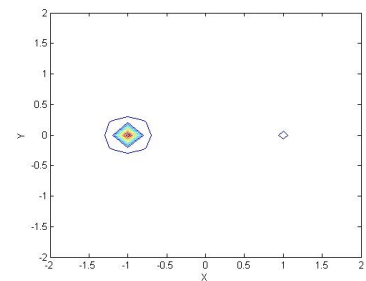

(e) Q-Criterion

Figure 4. Application of the VORTFIND algorithm with 8 sectors to two potential vortices of opposite direction and differing strength with a perpendicular uniform flow, grid size $21 \times 21$ nodes.

equations (12) in order to determine the mean Cartesian velocity field $\left(\overline{U_{i}}\right)$ and mean pressure $(\bar{P})$ of the flow:

$$
\begin{gathered}
\frac{\partial \overline{U_{i}}}{\partial x_{i}}=0 \\
\rho \frac{\partial \overline{U_{i}}}{\partial t}+\rho \frac{\partial \overline{U_{i} U_{j}}}{\partial x_{j}}=-\frac{\partial \bar{P}}{\partial x_{i}}+\frac{\partial}{\partial x_{j}}\left\{\mu\left(\frac{\partial \overline{U_{i}}}{\partial x_{j}}+\frac{\partial \overline{U_{j}}}{\partial x_{i}}\right)\right\}-\rho \frac{\partial \overline{u_{i}^{\prime} u_{j}^{\prime}}}{\partial x_{j}}+f_{i}
\end{gathered}
$$

The RANS equations are implemented in the commercial CFD code ANSYS CFX 11 (CFX) [29]. The governing equations are discretised using the finite volume method. The high-resolution advection scheme was applied for the results presented which varies between first and second order accuracy depending on spatial gradient. For a scalar quantity, $\phi$ the advection scheme is written in the form

$$
\phi_{i p}=\phi_{u p}+b \nabla \phi R
$$


where $\phi_{i p}$ is the value at the integration point, $\phi_{u p}$ is the value at the upwind node and $R$ is the vector from the upwind node to the integration point. The model reverts to first order when $b=0$ and is a second order upwind biased scheme for $b=1$. The high resolution scheme calculates $b$ using a similar approach as that given by [30], which aims to maintain $b$ locally to be as close to one as possible without introducing local oscillations. Collocated (non-staggered) grids are used for all transport equations, and pressure velocity coupling is achieved using an interpolation scheme based on that proposed by [31]. Gradients are computed at integration points using tri-linear shape functions defined in [29]. The linear set of equations that arise by applying the Finite Volume Method to all elements in the domain are discrete conservation equations. The system of equations is solved using a coupled solver and a multigrid approach.

\subsection{Case Study 1 - Body vortices around a manoeuvring submarine body}

The first case shows the use of the VORTFIND algorithm to post process the path of body vortices evolving around a manoeuvring submarine using two turbulence closure models.

Axis-symmetric bodies yawed to the free stream flow, such as an underwater vehicle in a turning manoeuvre experience complex three dimensional flows, see Figure 5, featuring; stagnation flow, a highly 3D boundary layer under the influence of strong pressure gradients and streamline curvature and the evolution and formation of free vortex sheets which roll-up to form streamwise body vortices. These body vortices result in a nonlinear increase in lift with increasing incidence angle, due to the low pressure associated with the core of the body vortex, which is impressed upon the nearby body surface.

RANS simulations have been performed using a 10M element structured mesh [9] to replicate the experimental data of Lloyd and Campbell [32]. They performed towing tank and rotating arm experiments on a $5 \mathrm{~m}$ long representative submarine body, designation DOR at the UK, Admiralty Research Establishment. The axi-symmetric body comprises an ellipsoid bow moving to parallel midbody with a fineness ratio (length/diameter) of 8.5 , with moves to a tapered stern section. Tests were performed at a diameter based Reynolds number of $1.3 \times 10^{6}$, which corresponds to 


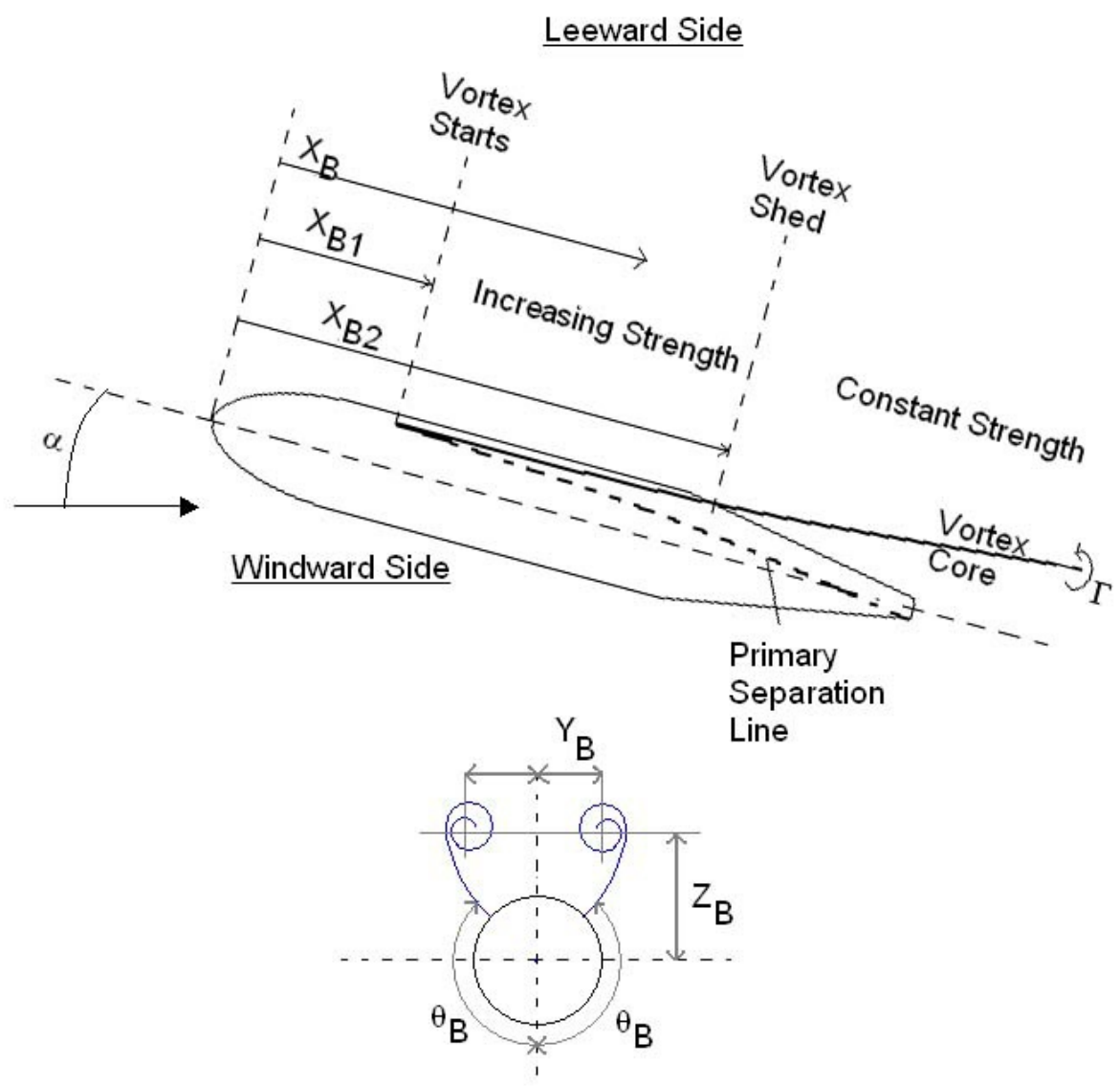

Figure 5. Body vortex notation, adapted from [32].

a length based Reynolds number of $11 \times 10^{6}$. The vorticity surrounding the body was measured using a Freestone vorticity probe [33]. Since uncertainty in the solution can also be attributed to the turbulence model, results for two turbulence models are presented.

1. Standard $k-\varepsilon$ with scalable wall functions.

2. SSG Reynolds Stress Model (SSG RSM) with scalable wall functions.

The improved VORTFIND algorithm is used to extract the path of the body vortices by analysing the transverse flow in body fitted co-ordinates at a series of transverse stations along the body, see Figure 6. Transverse planes were considered to test the improved VORTFIND's ability to identify hull vortices, resulting from crossflow separation over the hull at moderate drift angles. Experimentally the structure and path of the vortex was extracted using a Freestone probe. The 
output from this when suitably processed gives a direct measure of the vorticity. Hence it was possible to extract the vortex peak location without mapping the entire flow. Lloyd and Campbell [32] note that because the levels of vorticity are low, inaccuracies in the Freestone probe caused some uncertainty regarding the extent of the vortex and the value of the measured peak.

In the $Y_{B}$ direction the vortex tracks from the two turbulence models, see Figure 7 are relatively consistent and match quite closely to the vortex probe results. In the $Z_{B}$ direction the predicted vortex tracks from the two TCMs are similar up to $X_{B} / L=0.7$ which corresponds to the limit of the parallel midbody. As the hull tapers at the stern, the experimental results show the vortex core convecting downstream parallel to the hull centreline. However, the vortex tracks from the numerical results follow the curvature of the hull to varying degrees. 


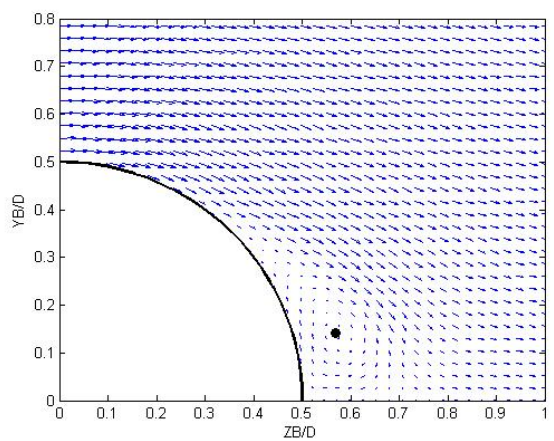

(a) $k-\varepsilon X_{B} / L=0.5$

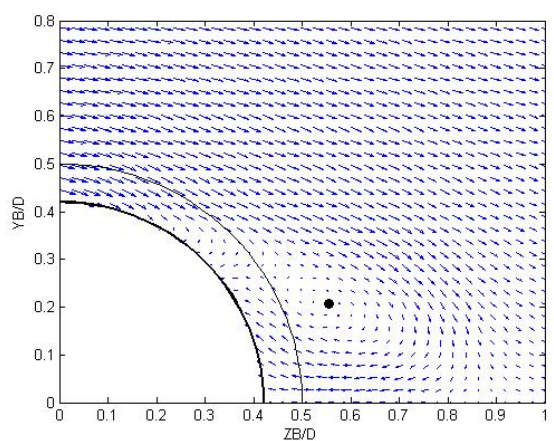

(c) $k-\varepsilon X_{B} / L=0.8$

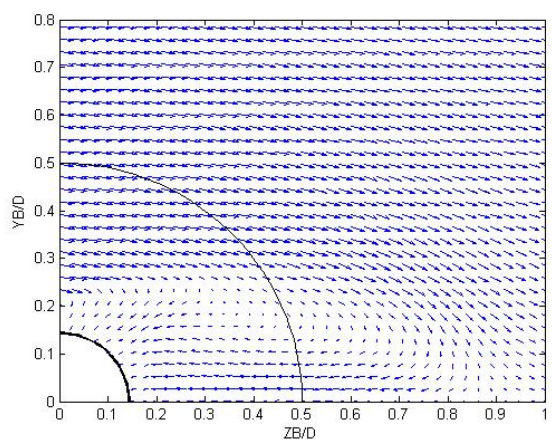

(e) $k-\varepsilon X_{B} / L=0.925$

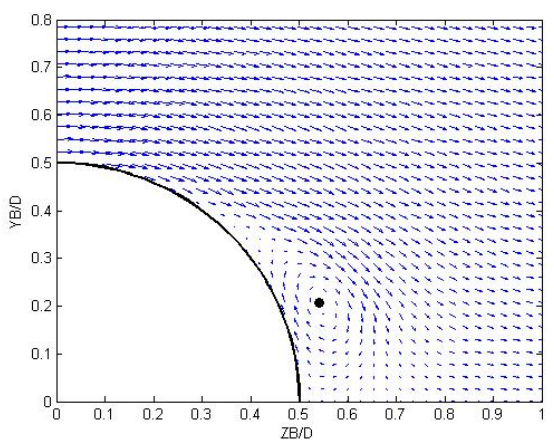

(b) SSG RSM $X_{B} / L=0.5$

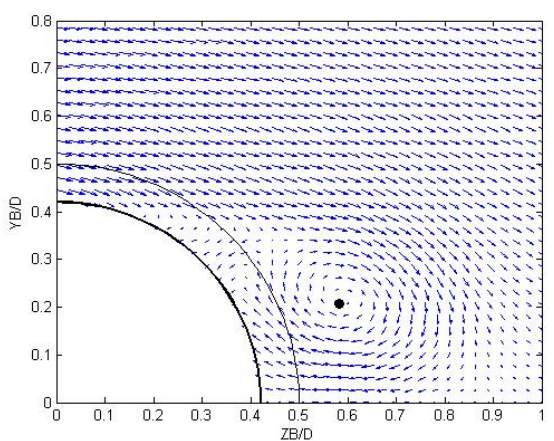

(d) SSG RSM $X_{B} / L=0.8$

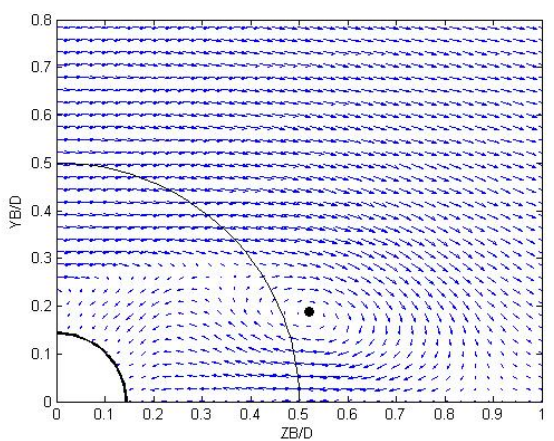

(f) SSG RSM $X_{B} / L=0.925$

Figure 6. Transverse velocity vectors at stations along the Body, thin black line represents maximum body diameter, thick black line represents local body diameter. Black spot marks vortex centre located by improved VORTFIND algorithm. 

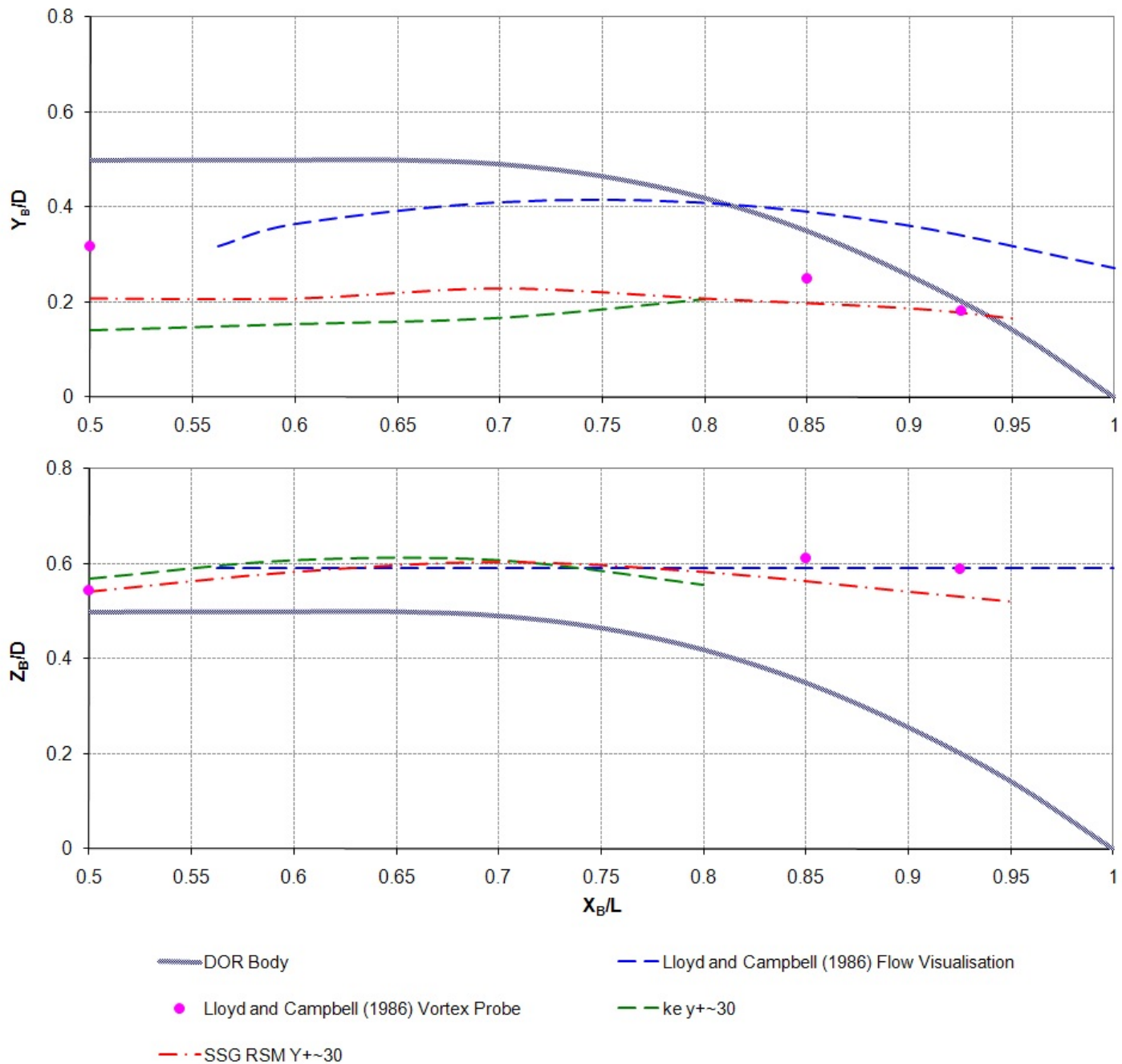

Figure 7. Comparison of estimated vortex centres from experimental and numerical results 


\subsection{Case Study 2 - Flow Downstream of a Propeller Model and Rudder}

The second case study demonstrates how the improved VORTFIND algorithm may be used as part of an iterative meshing strategy to improve spatial capture of a complex flow containing an unknown number of vortices.

Ship rudders are almost always placed downstream of the propeller so they can take advantage of the increased local velocity due to the presence of the propeller race. The flow around a rotating propeller is a highly complex and thus modelling a rotating propeller explicitly leads to a significant computational cost within a RANS simulation [34]. This is due to the high mesh resolution required around the blade to capture the flow features and the small time steps required to capture the transients flow features.

For work not concentrating on the propeller itself but rather the interaction of the propeller hull and rudder system it is not vital to capture all aspects of the propeller flow. As a result of this several body force propeller models have proposed to reduce computational cost $[35,36]$. When using a body force model the propeller is not physically represented by its geometry. Instead the effect of the propeller is included by representing the propeller as a series of axial and momentum source terms, $f b_{x}$ and $f b_{\theta}$ respectively, which are distributed over the propeller disc, these induce an axial and swirl acceleration in the fluid, representative of the action of the propeller. The case discussed here uses a prescribed distribution of thrust and torque in the radial direction [37] Further details of the body force propeller models are discussed in [38].

These approaches neglect blade frequency generated flow features and circumferentially average the action of the blade to provide a steady state model of the action of the propeller. However when placed upstream of a rudder the resulting flow is still highly complex, the physical boundary of the rudder splits the propeller race in two. The swirl component imparted by the propeller model leads to the two halves of the race rolling up to form vortical structures which interact with the rudder tip vortex, leading to a complex flow comprising of multiple vortex structures.

This example uses an iterative approach to mesh generation to capture the path of these multiple vortical structures as they convect downstream. 
The numerical simulations are based on wind tunnel tests performed by Molland and Turnock in the University of Southampton $3.5 \times 2.5 \mathrm{~m}$ RJ Mitchell Wind Tunnel, $[39,40]$. The experimental set up is shown in Figure 8. It comprise of a $1 \mathrm{~m}$ span, 1.5 geometric aspect ratio rectangular planform square tipped rudder (No. 2) with a constant NACA0020 section, and a representative $0.8 \mathrm{~m}$ diameter propeller based on the Wageningen B4.40 series placed at $X / D=0.39$, where $X$ is the distance between the propeller plane of revolution and the leading edge of the rudder at zero degrees incidence. Further details of the experiment can be found in [41].

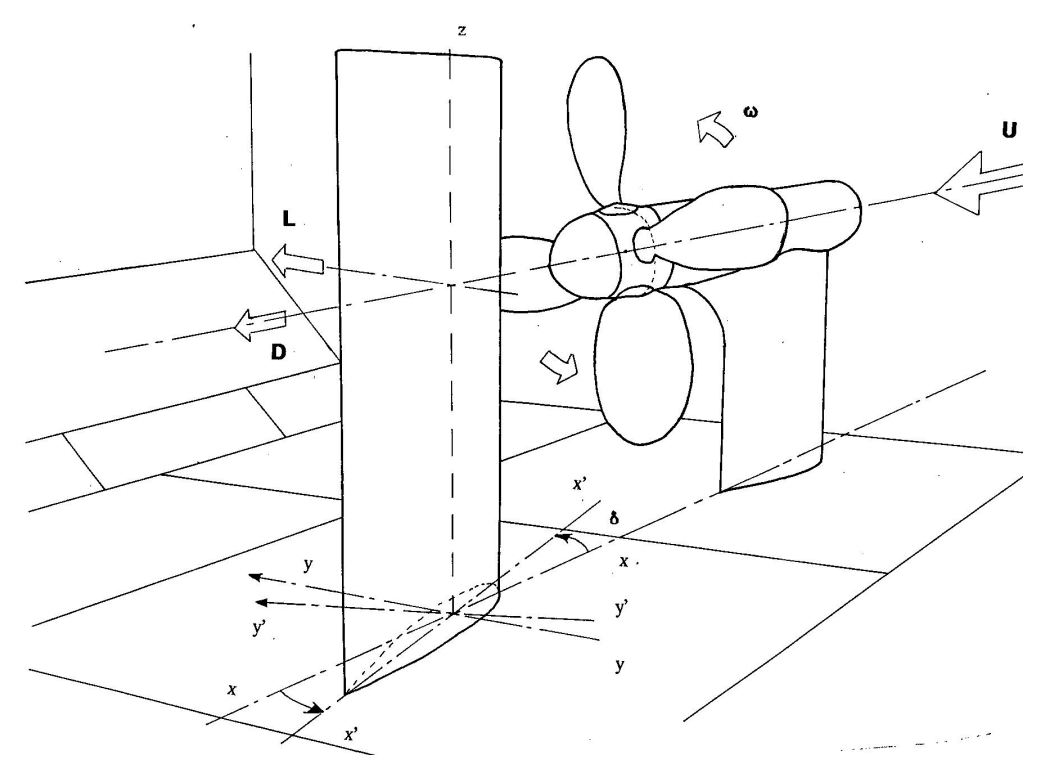

Figure 8. Isometric view of experimental setup [42]

Figure 9 illustrates the downstream mesh refinement process on a single 2D plane at the outlet of the domain (seven diameters downstream of the propeller plane $X / D=7$ ), a similar procedure was applied at six intermediate planes between the trailing edge and the outlet. The initial coarse mesh results in highly diffused flow structures. The tip vortex is evident in the lateral vector plot but none of the secondary vortices have propagated this far downstream $X / D=7$. However the initial mesh provides a good first estimate of the horizontal and vertical extents of the propeller race, taken as a box containing all nodes with axial velocities greater than the inflow velocity (shown as a box on the axial flow plots). The second mesh is refined in the region of the propeller race identified 
on the initial mesh. The 3D flow field derived from the second mesh is then post processed to identify not only the extents of the propeller race but the location of any vortex structures using the improved VORTFIND algorithm (highlighted as red dots on the transverse velocity vector plots).

The path of the vortices through the domain are tracked between the series of planes, and further mesh refinement is applied in this regions, the path of the vortices can be seen in the final mesh as darker circular regions.

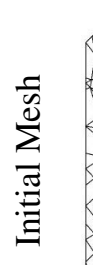
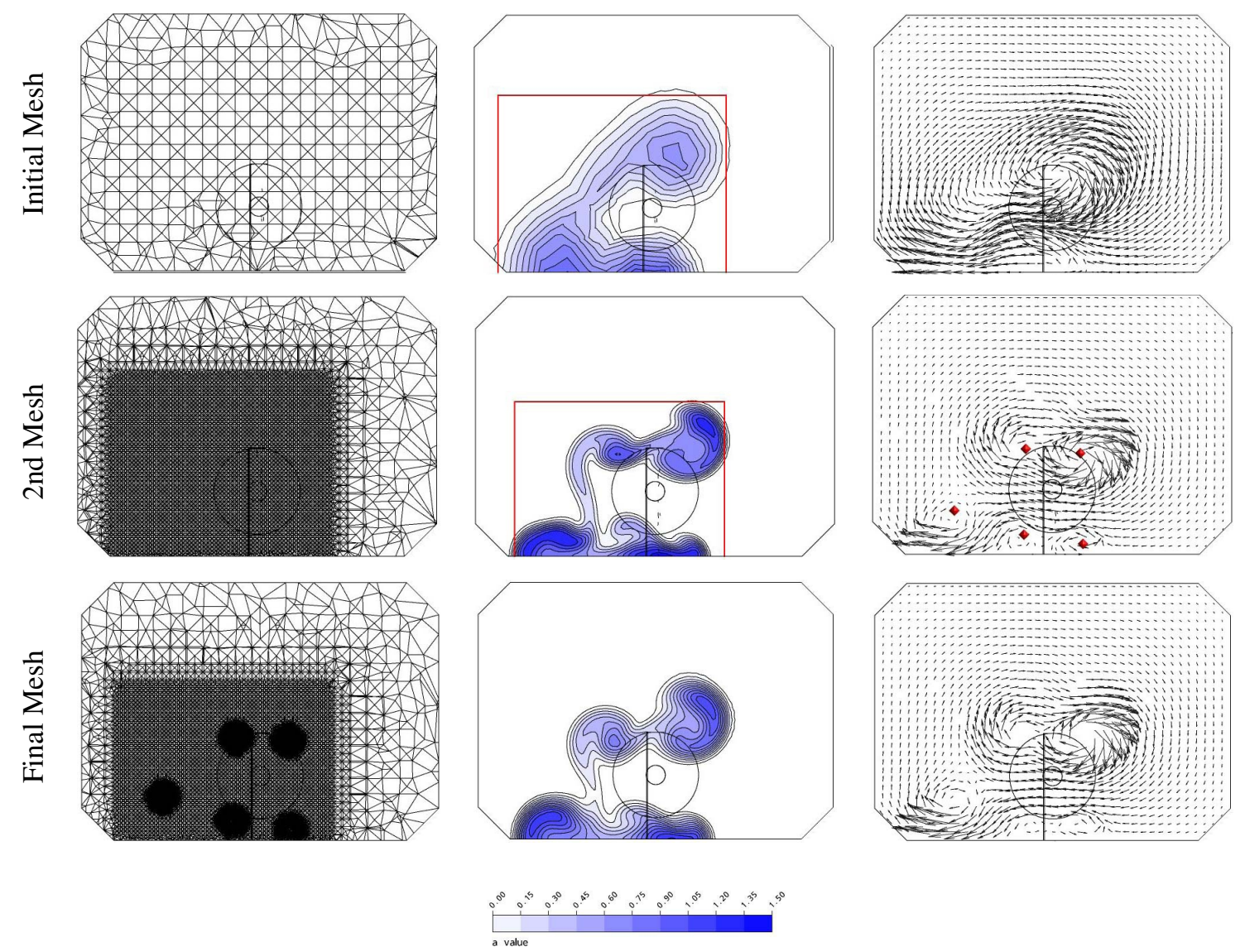

Figure 9. Example downstream mesh strategy. Case - Hough and Ordway rudder at $10^{\circ}$ incidence, $J=0.34$ $X / D=7$ at the domain outlet. Mesh (left), axial flow factor (centre) and transverse velocity vectors (right). Vortex locations identified by the improved VORTFIND algorithm marked by red diamonds and the red box describes the lateral and vertical extents of the propeller race. This information is then used to help define refinement regions for the proceeding mesh 


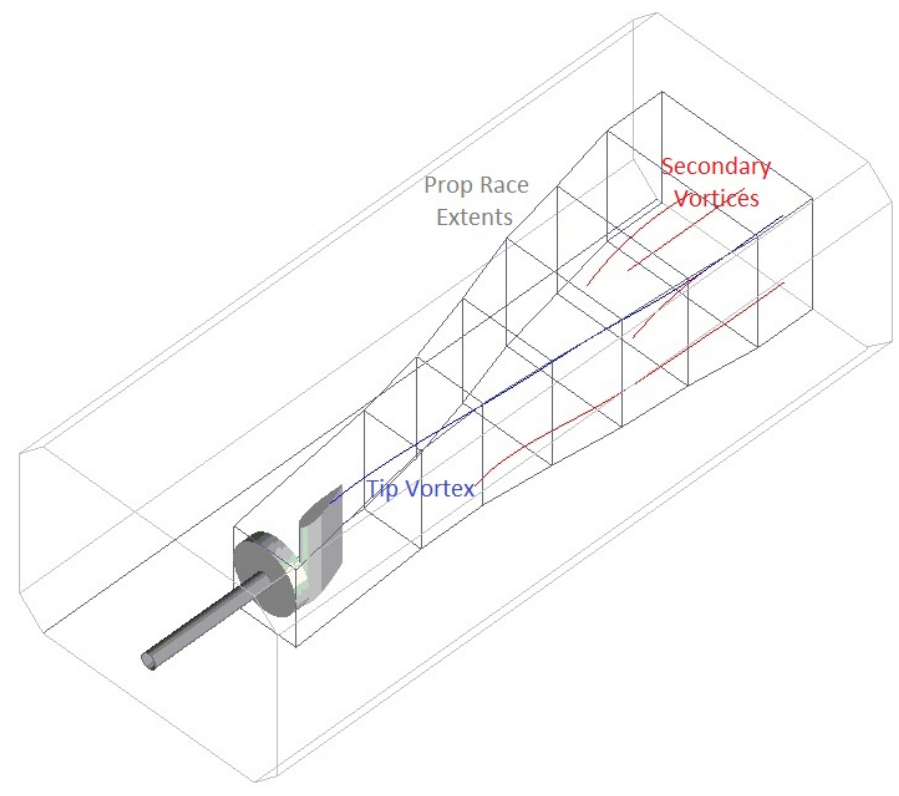

Figure 10. Isometric view of mesh domain for final mesh iteration, highlighting regions for mesh refinement in propeller race, and vortex core locations

Figure 10 highlights the refinement regions of the final mesh over the whole domain. Capture of the propeller race was typically well achieved with 50 mesh grid points along its lateral extents, with the same mesh density longitudinally. However, this density while suitable for capturing the higher axial velocity was unable to resolve the full swirling behaviour of the vortex effected regions. The rudder tip vortex is assumed to initiate at 2/3rds chord on the suction side of the rudder tip and extends downstream through the extent of the domain. Additional secondary vortex structures form downstream as the swirl from the propeller rolls up into coherent structures under the influence of the stronger tip vortex. Capture of the vortex core region typically requires at least 15 elements across the vortex core (more are required when using unstructured tetrahedral meshes due to the associated numerical diffusion). Additional local refinement was implemented on the rudder surface and in development prismatic elements of the rudder surface to capture the boundary layer.

Improved spatial resolution in and around the vortices significantly modifies the the shape of the propeller race. From a weakly defined region of flow at a higher velocity to free stream on the initial 
mesh the final mesh provides a clearly defined accelerated flow region with clear and well defined vortical structures

\section{CONCLUSIONS}

Spatial resolution of vortical structures around manoeuvring marine vehicles is vital to ensure correct modelling of the pressure field surrounding the vehicle which plays a major role in determining the hydrodynamic forces and moments acting on the vehicle. The improved VORTFIND algorithm is a robust and computationally inexpensive method of identifying the vortex core centre using just the velocity data on a transverse plane. Vortex core lines may be generated from a series of line segments passing through the local extreme of a series of planes. It is a line method that although not strictly Galilean invariant, allows multiple vortices to be captured, only requiring knowledge of the velocity field, as opposed to the above methods which require further details such as grid connectivity or velocity gradients and usually exhibit poor performance on the initial coarse mesh required to initiate the adaptation cycle.

The method has been improved to make it applicable for use with complex flows with multiple vortex structures, providing a fast and robust algorithm for identifying vortex paths from either experimentally or numerically generated velocity fields, independent of vortex strength. The approach may be run as either as post processing or built into the RANS simulation itself to allow inline iterative mesh adaption, where its ability to identify vortices on even very coarse meshes is highly advantageous.

\section{ACKNOWLEDGEMENT}

Dr Phillips' PhD studentship was jointly financed by the School of Engineering Science and the National Oceanography Centre, Southampton.

REFERENCES 
1. Lee S, Jin E, Lee H. Evaluation of the vertical plane stability by CFD. Proceedings of the fifteenth(2005) International Polar Engineering Conference, Seoul Korea, 2005.

2. Wu B, Xing F, Kuang X, Mia O. Investigation of hydrodynamic characteristics of submarine moving close to the sea bottom with CFD methods. Journal of Ship Mechanics 2005; 9:14-17.

3. Bellevre D, de Tuesta AD, Perdon P. Submarine manoeuvrability assessment using computational fluid dynamic tools. Proc. 23th Symposium on Naval Hydrodynamics, Val de Reuil, France, 2000; 820-832.

4. Boger D, Dreyer J. Prediction of hydrodynamic forces and moments for underwater vehicles using overset grids. Proceedings of AIAA Aerospace Meeting and Exhibit Reno, Nevada, Jan 9-12, 2006.

5. Turnock S, Pashias C, Rodgers E. Flow feature identification for capture of propeller tip vortex evoloution. Proceedings of the 26th symposium on naval hydrodynamics, Rome, Italy, 2006; 223-240.

6. Kasmai N, THompson D, Luke E, Jankun-Kelly M, Machiraju R. Feature-based adaptive mesh refinement for wingtip vortices. International journal for numerical methods in fluids 2010; 66:1274-1294.

7. Rhee S, Hyung S, Joshi S. Computational validation for flow around a marine propeller using unstructured mesh based navier-stokes solver. SME International Journal Series B 2005; Vol. 48, No. 3:pp.562-570.

8. Bensow R, Bark G. Implicit les predictions of the cavitating flow on a propeller. Journal of Fluids 2010; 132:041302.

9. Phillips A, Turnock S, Furlong M. Influence of turbulence closure models on the vortical flow field around a submarine body undergoing steady drift. Journal of Marine Science and Technology 2010; 15:201-217.

10. Jiang M, Machiraju R, Thompson D. The Visualization Handbook, chap. Detection and Visulalization of Vortices. Academic Press, 2004; 295-309.

11. Kolar V. Vortex identification: New requirements and limitations. International Journal of Heat and Fluid Flow 2007; 28:638-652.

12. Hunt J, Wray A, Moin P. Eddies, stream and convergence zones in turbulent flows. Technical Report, Center for Turbulence Research Report CTR-S88 1988.

13. Levy Y, Degani D, Seginer A. Graphical visualization of vortical flows by means of helicity. AIAA Journal 1990; 28:1347-1352.

14. Berdahl C, Thompson D. Eduction of swirling structure using the velocity gradient tensor. AIAA Journal 1993; 31:97-103.

15. Jeong J, Hussain F. On the identifcation of a vortex. Journal of Fluid Mechanics 1995; 285:69-94.

16. Banks D, Singer B. A predictor-corrector technique for visualizing unsteady flow. IEEE Trans. on Visualization and Computer Graphics 1995; 1:151-163.

17. Sujudi D, Haimes R. Identification of swirling flow in 3D vector fields. AIAA 12th Computational Fluid Dynamics Conference, Paper 95-1715, 1995.

18. Roth M, Peikert R. A higher-order method for finding vortex core lines. In IEEE Visualization '98, October, 1998.

19. Strawn R, Kenwright D, Ahmad J. Computer visualization of vortex wake systems. AIAA Journal 1999; 37:511512. 
20. Sadarjoen IA, Post FH, Ma B, Banks DC, Pagendarm HG. Selective visualization of vortices in hydrodynamic flows. In IEEE Visualization '98, pages 419-422, October, 1998.

21. Jiang M, Machiraju R, Thompson D. A novel approach to vortex core region detection. Joint Eurographics IEEE TCVG Symposium on Visualization, pages 217-225, 2002.

22. Pemberton R, Turnock S, Dodd T, Rogers E. A novel method for identifying vortical structures. Journal of Fluids and Structures 2002; 16:1051-1057.

23. Lugt H. Vortex flow in nature and technology. New York, John Wiley \& Sons, 1983.

24. Pashias C. Propeller tip vortex simulation using adaptive grid refinement based on flow feature identification. PhD Thesis, University of Southampton 2005.

25. Pemberton R, Turnock S, Rogers E. Identification of bilge vortices within cfd simulations. The Transactions of The Royal Institution of Naval Architects, International Journal of Maritime Engineering 2003; 145:57-56.

26. Turnock S, Querard A. Measurements of the evolution of the wake and tip vortex of a control surface undergoing periodic motion. 27th American Towing Tank Conference, St. John's, Canada, 06 - 07 Aug, 2004; 1-19.

27. Pemberton R. A vortex identification technique for grid adaptation. PhD Thesis, School of Engineering Sciences, University of Southampton. 2003.

28. Yin X, Germay N. a fast genetic algorithm with sharing scheme using cluster methods in multimodal function optimisation. Proc. of the Int. Conf. on Artificial Neural Nets and Genetic Algorithms, 1993; 450-457.

29. ANSYS. ANSYS CFX, Release 11.0. ANSYS 2006.

30. Barth T, Jesperson D. Design and application of upwind schemes on unstructured meshes. AIAA Paper 89-0366 $1989 ;$.

31. Rhie $\mathrm{C}$, Chow W. A numerical study of the turbulent flow past an isolated airfoil with trailing edge separation. AIAA Paper 82-0998 1982; .

32. Lloyd A, Campbell I. Experiments to investigate the vortices shed from a submarine -like body. 59th meeting of the AGARD fluid Dynamics Panel Symposium, 1986.

33. Freestone M. Vorticity measurement by a pressure probe. Aeronautical Journal 1988; 92:29-35.

34. Phillips AB. Simulations of a self propelled autonomous underwater vehicle. PhD Thesis, School of Engineering Sciences, University of Southampton 2010.

35. Schetz J, Favin S. Numerical solution for the near wake of a body with a propeller. Journal of Hydronautics 1977; 11:136-141.

36. Stern F, Kim H, Patel N, Chen H. A viscous-flow approach to the computation of propeller-hull interaction. Journal of Ship Research 1988; 32:246-262.

37. Hough G, Ordway D. The generalised actuator disc. Developments in Theoretical and Applied Mechanics 1965; 2:317-336.

38. Phillips A, Turnock S, Furlong M. Accurate capture of propeller-rudder interaction using a coupled blade element momentum-rans approach. Ship Research Technology schiffstecnik 2010; 57:128-139. 
39. Molland A, Turnock S. Influence of propeller loading on ship rudder performance. Transactions of the Royal Institution of Naval Architects 1992; 135:105-120.

40. Molland A, Turnock S, Smithwick J. Wind tunnel tests on the influence of propeller loading and the effect of a ship hull on skeg-rudder performance. Technical Report, University of Southampton, Ship Science Report 901995.

41. Molland A, Turnock S. Marine Rudders and Control Surfaces. Butterworth-Heinemann, 2007.

42. Molland A, Turnock S. Wind tunnel investigation of the influence of propeller loading on ship rudder performance. Technical Report, Ship Science Report No. 461991. 